\title{
Erratum to: Adaptive Matrices and Filters for Color Texture Classification
}

\author{
Ioannis Giotis • Kerstin Bunte • Nicolai Petkov • \\ Michael Biehl
}

Published online: 26 October 2013

(C) Springer Science+Business Media New York 2013

\section{Erratum to: J Math Imaging Vis (2013) 47:79-92 DOI 10.1007/s10851-012-0356-9}

The following passages were not properly cited in the original paper. The authors apologize for this error and would like to update the paper accordingly.

On page 2, lines 174-176 should read as: "The original LBP operator [23] creates labels for the image pixels by thresholding their $3 \times 3$ neighborhood with the center value" [36].

And lines 187-198 should read as: As pointed out in [35]: "another extension to the original operator is the definition of the so called uniform patterns, which can be used to reduce the length of the feature vector and implement a simple rotation-invariant descriptor. This extension was inspired by the fact that some binary patterns occur more commonly in texture images than others. A local binary pattern is called uniform if it contains at most two transitions from 0 to 1 or vice versa when it is traversed circularly. Ojala et al. [24] noticed in their experiments that uniform patterns account for a little less than $90 \%$ of all patterns when using a $(8,1)$ neighborhood and for around $70 \%$ with a $(16,2)$ neighborhood."

[35] Pietikäinen, M.: Local Binary Patterns, Scholarpedia 5(3), 9775 (2010).

[36] Hadid, A., Zhao, G., Ahonen, T., Pietikäinen, M.: Face analysis using local binary patterns. In: Handbook of Texture Analysis, pp. 347-373 (2007).

The online version of the original article can be found under doi:10.1007/s10851-012-0356-9.

I. Giotis $(\varangle) \cdot$ N. Petkov $\cdot$ M. Biehl

Johann Bernoulli Institute for Mathematics and Computer

Science, University of Groningen, PO Box 407, 9700 AK,

Groningen, The Netherlands

e-mail: i.e.giotis@rug.nl

K. Bunte

CITEC Center of Excellence-Cognitive Interaction Technology, Bielefeld University, Universitaetsstrasse 21-23, 33615, Bielefeld, Germany 